\title{
15 FACILITATING THE PUBLIC DISCUSSIONS OF ADVANCE CARE PLANNING WITH AUDIO-VISUAL MEDIA IN TAIWAN - A ACTION RESEARCH
}

\author{
10.1136/bmjspcare-2011-000053.15
}

W Yang ${ }^{1}$, C S Chao 2 , G Chiu ${ }^{3}$, P C Lin', Yih-Chyang Weng ${ }^{5}$, Y M Kuo ${ }^{6}$, Ya Lan Shih7, Wei-Shu Lai ${ }^{1}$ Department of Nursing, College of Medicine, National Cheng-Kung University, Tainan, Taiwan; ${ }^{2}$ National Cheng-Kung University Hospital Hospice Ward, Tainan, Taiwan; ${ }^{3}$ National Cheng-Kung University Medical Institute and Hospital, Tainan, Taiwan; ${ }^{4}$ Nantou Hospital of Health of Department, Nantou, Taiwan; ${ }^{5}$ Department of Electrical Engineering, National Cheng-Kung University, Tainan, Taiwan; ${ }^{6}$ Hospice Unit, National Cheng-Kung University Hosptial,Tainan,Taiwan; IInstitute of Allied Health Sciences, College of Medicine, National Cheng-Kung University, Tainan, Taiwan; ${ }^{8}$ Department of Nursing, Meiho University, PingTung, Taiwan

With the action research method, the senior physicians and nurses became involved with the community: they interviewed 32 seniors, ages 50 to 80 , through a focus group to understand their needs and suggestions regarding issues related to terminal medical decisions. The following five points regarding the presentation are summarized: (1) be easy to understand, not boring; (2) be realistic and accurate; (3) clearly guide the thinking of the important issues; (4) narrative; (5) present with actual images. The physicians and nurses further worked with the IT professionals. After five panel meetings and three times modified according to the experts and audiences' suggestions, a 10-min video that combines flash design and real images was created. Total 291 valid questionnaires were collected in a university, a church and a nursing home; the participants included elementary school students as well as $80+$ years old seniors. A discussion about the terminal medical decision was guided after each play of the video. $97.6 \%$ expressed interest in the issue related to terminal medical decision and the willingness of sharing and discussing it with their families. 98.3\% indicated that when they or their illness reached the final stage, they wanted no more CPR and wished to receive hospice care. The results show the effect to use audio-visual media to guide the public to understand what is actually happening in terminal emergency treatment, to think about their own terminal medical decisions and to stand up and defend their own medical autonomies. 